\title{
EL DEBATE SOBRE EL OMNIVORISMO CULTURAL Una aproximación a nuevas tendencias en sociología del consumo
}

\author{
THE DEBATE ON CULTURAL OMNIVOROUSNESS \\ New trends in sociology of consumption \\ Carlos Jesús Fernández Rodríguez carlos.fernandez@uam.es \\ Universidad Autónoma de Madrid. España
}

RIIE HEIKKILÄ riie.heikkila@helsinki.fi

Universidad de Helsinki. Finlandia

\begin{abstract}
Resumen
En las últimas décadas uno de los debates más interesantes en el campo de la sociología del consumo ha sido la cuestión del omnivorismo cultural, planteada generalmente como una crítica a la teoría de la distinción establecida por Bourdieu en sus trabajos más clásicos. Frente a la idea de diferenciación y jerarquización de los estilos de vida basada en la adquisición de capital cultural que enunciaba este autor, los defensores del omnivorismo cultural defienden por el contrario que los gustos legítimos de las nuevas clases dominantes se caracterizan, en la actualidad, por un amplio abanico de preferencias culturales, con gustos que se extienden desde las artes más refinadas a las manifestaciones propias de subculturas populares. El objetivo de este artículo es el de realizar un repaso de la teoría del omnivorismo cultural, prestando atención a las líneas principales del debate que ha generado y las principales críticas que ha suscitado.
\end{abstract}

\section{Palabras Clave}

Distinción, Gustos culturales, Omnívoro cultural, Sociología del consumo.

\begin{abstract}
During the last decades, one of the most interesting debates in the field of sociology of consumption has been the issue of cultural omnivorousness, usually outlined as a critique towards Bourdieu's classic distinction theory. As opposed to the idea of differentiation and hierarchization of lifestyles based on the acquisition of cultural capital, the advocates of cultural omnivorousness claim that the new legitimate taste of the new ruling classes is depicted by a greater breadth of cultural preferences, whose range varies from the most refined arts to others more typical of popular culture. The aim of this article is to review the cultural omnivorousness theory as well as the main trends in the debate that has helped to launch. The main critiques that the concept of cultural omnivore has received by some authors will not be overlooked.
\end{abstract}

\author{
KEYWORDS \\ Cultural Omnivorous, Cultural Tastes, Distinction, Sociology of Consumption.
}




\section{INTRODUCCIÓN ${ }^{1}$}

Un tema central en la sociología del consumo es, sin duda alguna, la preocupación por la dimensión simbólica del mismo. El consumo juega un papel muy importante en la estructuración de nuestra identidad personal y en las formas que tenemos de relacionarnos con aquellos que nos rodean (Alonso 2005; Douglas e Isherwood 1979).

En el caso del consumo de productos culturales (música, literatura, arte, cine), dicha dimensión simbólica ha jugado un papel especialmente importante desde el momento que sirven de elemento clave para estrategias tanto a favor de la elevación social de los individuos, como de mantenimiento de las condiciones de la reproducción social, dentro de un contexto de sociedad de clases (Callejo 1995). Sean estas estrategias conscientes 0 inconscientes (Daloz 2009), lo cierto es que han favorecido la segmentación de las prácticas de consumo tanto en la etapa histórica de la consolidación de la norma de consumo de masas, como en la posterior reorganización económica posfordista, que se caracterizaría por nuevas dinámicas de fragmentación, individualización, virtualización y globalización (Alonso 2005).

El debate sobre el modus operandi de estas estrategias de distinción tuvo un hito intelectual en la publicación del influyente trabajo de Pierre Bourdieu La distinción (1998; edición original de 1979). Bourdieu sienta en este trabajo las bases para una teoría explicativa del gusto basada en la división de clases que recoge, por primera vez, una teoría que permite conceptualizar las relaciones entre clase social, estatus y gusto (Peterson 2005). Para Bourdieu, las prácticas de consumo están fuertemente influidas por la clase social objetiva a la que pertenece el individuo, y en la que se ha configurado un sistema de disposiciones (el habitus) que genera un conjunto de condicionamientos en relación a (entre otros muchos comportamientos) las pautas de desarrollo de los gustos, que se ven así modelados por la clase social de origen. Los marcos de referencia del espacio del consumo serían así tres: uno estructural (la clase social), otro simbólico (el estilo de vida), y el habitus. Las diferencias en los gustos y los correspondientes estilos de vida asociados serían, así, consecuencia de las desigualdades sociales, y se basarían en estrategias de distinción operadas sobre la base de una lógica de la dominación. Se establecería, así, una homología entre el campo de las relaciones sociales y el del consumo cultural, por la que los distintos actores sociales tendrían un abanico de aficiones y preferencias limitado y fuertemente constreñido por sus orígenes de clase (Bourdieu 1983). Esto implicaría la formación de un gusto legítimo (y por tanto una legitimidad cultural) propio de las clases dominantes, caracterizado por una distinción excluyente respecto a unos gustos populares estigmatizados por dichas élites como vulgares (que

\footnotetext{
${ }^{1}$ Este artículo se ha realizado gracias al proyecto de investigación del Ministerio de Ciencia e Innovación, con referencia CSO2008-02886. Asimismo, ha contado con apoyo del proyecto "Cultural Capital and Social Diferentiation in Contemporary Finland: An International Comparison", con referencia SA 2006-1114819.
} 
de hecho se estigmatizan, por parte de los observadores de élites y clases medias, como ajenos a la propia idea de cultura) ${ }^{2}$.

Sin embargo, y como varios autores han apuntado (Ariño 2007; Noya 1998), el esquema conceptual de Bourdieu ha sido puesto en cuestión en las últimas dos décadas en el terreno de la sociología y los estudios culturales, dentro de un debate más amplio sobre la conformación social de los gustos (Warde, Wright y Cayo-Gal 2007). Una de las corrientes teóricas que ha realizado esta labor de crítica de forma más sistemática ha sido la del omnivorismo cultural ${ }^{3}$, que ha contribuido, sin lugar a dudas, a generar uno de los debates más interesantes en la sociología del consumo actual. El término omnívoro, derivado del latín omnivorus (aquel que devora todo), y que se ha convertido en una categoría de clasificación zoológica (aquellos animales que se alimentan tanto de plantas como de otros animales), tiene otra definición posible (al menos en inglés): la de alguien que es capaz de asimilar casi todo, fundamentalmente a un nivel mental o intelectual ${ }^{4}$.

El modesto objetivo de este artículo no es otro que el de realizar una presentación y discusión de las últimas novedades en este debate. Comenzaremos describiendo el origen del concepto de omnivorismo cultural para, desde ahí, analizar los principales argumentos de dicho planteamiento teórico y presentar las principales críticas al mismo, a la vez que se discutirán posibilidades futuras de investigación en este terreno desde una perspectiva metodológica cualitativa. El texto se cerrará con una breve conclusión.

\section{EL CONCEPTO DE OMNIVORISMO CULTURAL: ORIGEN Y DEBATES SOBRE LA CUESTIÓN}

Como se ha apuntado en la Introducción, la teoría del omnivorismo cultural nace como una reacción a la hegemonía que, en los estudios culturales y la sociología del gusto, estaba disfrutando la teoría de Bourdieu sobre la distinción, ya citada anteriormente. No vamos a realizar aquí un repaso de esta última en profundidad, ya que existen trabajos especializados que repasan las principales contribuciones teóricas de este gigante de la sociología mundial (Alonso, Martín Criado y Moreno Pestaña 2004). No obstante, sí es conveniente resaltar algunos aspectos de la misma, que serán confrontados por los teóricos del omnivorismo cultural, y que se señalan a continuación:

a) La teoría de Bourdieu, como se ha comentado en la introducción, afirma la estrecha relación entre la clase social y la formación del gusto, que influiría en la constitución de un abanico limitado de preferencias culturales. Ello se debe, en buena medida, al

\footnotetext{
${ }^{2}$ Para profundizar en esta cuestión recomendamos la lectura del capítulo sobre Bourdieu, en Alonso (2005).

${ }^{3}$ Antonio Ariño (2007) ha traducido este término como "omnivoridad cultural".

${ }^{4}$ Un lector omnívoro, así, sería capaz de degustar con igual fruición literatura clásica legitimada por el mundo de la crítica o la academia junto a géneros considerados menores como las novelas de ciencia-ficción.
} 
conocido habitus, conjunto de disposiciones que estructuran la mirada del individuo, incorporando a ésta los efectos de las prácticas sociales.

b) Bourdieu atribuye a los individuos la posesión de un capital social, económico y cultural que define la posición de una persona en la jerarquía social, y observa que los individuos los utilizan de formas muy distintas dependiendo de si pertenecen a las élites económicas o culturales. Así, los individuos con elevado capital económico parecen mostrar un interés por los automóviles y otros bienes suntuosos que no se corresponde con los individuos con elevado capital cultural, más interesados en el arte o el disfrute de experiencias con un elevado componente estético. Para Bourdieu, el uso por parte de los miembros de las élites y clases altas de dichos capitales debe ser "consistente" para evitar confusiones y permitir su reconocimiento social.

c) Las personas tratan, dentro de los diferentes espacios sociales (campos), de acumular un capital determinado, que en el caso que nos ocupa sería el capital cultural, y cuya adquisición es fundamental para el reconocimiento social. Se realiza, por tanto, una importante distinción entre los gustos considerados legítimos, asociados a una idea de "alta cultura" que exige refinamiento, y otros gustos "vulgares" asociados a la cultura popular. Por ello, en su investigación aparece que las élites son las que definen el gusto legítimo; las clases medias tratan de acumular dicho capital cultural para favorecer su movilidad social ascendente, y se interesan por la música clásica o la pintura en un ejercicio de "buena voluntad" (aunque sin llegar a dominar totalmente el campo del gusto legítimo); las clases obreras, mientras tanto, disfrutan únicamente de manifestaciones diversas de la cultura popular.

d) En la distinción entre gustos subyace la lógica de dominación de clase existente en la sociedad, de la que las estrategias de acumulación de capital cultural no dejan de ser un ejemplo más. El modelo, según Bourdieu es, en gran medida, generalizable al resto de sociedades capitalistas avanzadas.

Sin embargo, y como varios autores (Ariño 2007; Noya 1998) han señalado, desde que Bourdieu recopiló los datos empíricos sobre los que construyó su teoría hasta los tiempos actuales se han producido unas importantes transformaciones en las sociedades occidentales, relacionadas con un cambio en el paradigma de regulación capitalista, con un giro desde la regulación fordista a un nuevo sistema de acumulación flexible denominado por muchos teóricos "posfordismo" (Harvey 1998). Acompañando a estas transformaciones, Lizardo y Skiles (2009) afirman que uno de los cambios recientes en el Occidente posindustrial que mejor ha sido documentado sería el del declive de un sistema de estratificación cultural rígido, sustituido por un nuevo modelo caracterizado por el fuerte contraste entre unas clases altas con un amplio abanico de gustos y unas clases bajas que apenas se interesan por la cultura, salvo por unas escasas manifestaciones populares.

La fragmentación social y el escenario de globalización económica han conducido, de esta forma, a una necesidad de replantear el análisis del consumidor y de sus formas de distinción simbólica. Surgen además nuevos tipos de consumidor cultural alejados del 
tradicional esnobismo de las clases altas, como son los cosmopolitas (Hannerz 1990), los BoBos (Brooks 2001) o el reciente Homo Sampler (Fernández Porta 2008), muchos de ellos pertenecientes a las nuevas clases creativas (Florida 2002) que pasan a ocupar las posiciones de clase medias y altas en la nueva sociedad de la información, y cuya aproximación al consumo cultural es más abierta y hedonista 5 . La distinción dualista entre alta cultura y cultura popular pierde parte de su fuerza como explicación de las pautas de consumo, ante un creciente eclecticismo en las prácticas de consumo cultural y el aumento de la tolerancia ante otras preferencias, además de unas fronteras más borrosas entre lo que se considera alta cultura y no; el sistema de clasificación y jerarquización de las artes se ha debilitado, creándose una nueva situación de "desclasificación" (Di Maggio 1987). Asimismo, y como Lamont (1992) señaló en una famosa investigación comparada entre Francia y Estados Unidos, el esnobismo cultural, aun siendo una tendencia presente en las grandes ciudades de ambos países, era únicamente un patrón hegemónico en París, no teniendo tanta influencia en las ciudades norteamericanas donde el capital económico o la honestidad personal eran factores más importantes en, por ejemplo, cuestiones como la selección de las amistades. Las fronteras, según Lamont, son más borrosas de lo que parecen. Por lo tanto, la aplicabilidad en otros contextos espaciales o sociohistóricos de la teoría bourdieuana se había empezado a poner en cuestión, como señalan algunos teóricos (Kane 2003). Estas dificultades preparan el terreno para nuevos enfoques como el del omnivorismo cultural.

Pero, ¿qué significa exactamente omnivorismo cultural? Una definición bastante ajustada sería la realizada por un grupo de investigación británico, en la que se especifica que la "tesis del omnivorismo" arguye que, en los países occidentales, existe un sector de la población al que le gusta un abanico mayor de formas de cultura que en épocas previas, lo que reflejaría un aumento de la tolerancia social hacia otras formas y gustos culturales que minaría, en cierto modo, las actitudes relacionadas con el esnobismo (Warde, Wright y Cayo-Gal 2007). Supone una alternativa a una teoría del gusto basada en el esnobismo como factor excluyente y distintivo (Bergham y van Eijck 2009; Peterson 1992).

En las ciencias sociales, la discusión sobre el omnivorismo cultural comenzó a gestarse en la década de los noventa, gracias, en buena medida, al trabajo del sociólogo norteamericano, recientemente fallecido, Richard Peterson. Los textos que, sin duda alguna, contribuyeron de forma decisiva a la popularización del término fueron dos trabajos suyos (Peterson y Simkus 1992; Peterson y Kern 1996). El planteamiento de Peterson era el de realizar una crítica a la concepción de distinción planteada por Bourdieu (1998), que había planteado la posesión de un gusto legítimo (concretado en el consumo de unos objetos simbólicos determinados y reconocibles) y la amplitud de conocimiento en el terreno de la cultura como elementos automáticamente vinculados a una posición

\footnotetext{
${ }^{5}$ Bourdieu (1998), no obstante, había predicho dicha evolución cuando estudiaba a las nuevas clases medias y su "ética de la diversión".
} 
de clase elevada. Para ello, los dos autores utilizaron un conjunto de encuestas realizadas en Norteamérica (en los años 1982 y 1992) para demostrar que, desde la década de los ochenta, se había ido desarrollando, entre las clases medias y altas norteamericanas, una tendencia a interesarse, cada vez más, por objetos de consumo cultural de las clases bajas. En los diez años que había pasado entre la realización de las dos encuestas, los autores comprobaron que dicha tendencia había crecido notablemente, lo que les había llevado a afirmar que el "omnivorismo cultural" estaba sustituyendo, de forma progresiva, un cierto elitismo intelectual y esnobismo que había sido frecuente entre las clases altas (frente a un "univorismo" o abanico estrecho de gustos que caracterizaría a las clases populares). Este análisis guardaba además cierta relación con la tesis de la "desclasificación artística" enunciada por Di Maggio (1987), en la que se señalaba que las barreras establecidas entre alta cultura y cultura popular se estaban erosionando en Estados Unidos, como resultado de varios factores: el ascenso de un cierto grupo de las clases medias, vinculado al mundo organizacional, a la élite nacional; la creciente influencia del criterio comercial en los principios de clasificación del arte, resultado del crecimiento de las industrias culturales (y que implica que por ejemplo la música pop-rock haya ganado respetabilidad); la emergencia de un campo de la alta cultura autónomo y altamente competitivo, que se desliga de algunas élites; y la influencia de la producción masiva de bienes culturales y de la participación del Estado moderno en las políticas culturales.

Años después, y con motivo de un extenso artículo retrospectivo en el que hacia una valoración de sus propias contribuciones y de las aportaciones de otros investigadores al desarrollo del debate sobre el omnivorismo cultural, Peterson (2005) reformuló algunos de sus conceptos clave. En su opinión, el trabajo que habia publicado previamente con Simkus (Peterson y Simkus 1992) adolecía de algunos defectos, como el de presentar un debate meramente unidimensional en el que, de manera simplificada, los esnobs se habían vuelto omnívoros frente a los consumidores más dejados (slobs) y menos interesados por adquirir un estatus simbólico, que se estancarían en una condición de unívoros. Mientras, en su artículo con Kern (Peterson y Kern 1996) había procedido a una reformulación de las categorías de clasificación, prestando más atención al abanico de los gustos: los omnívoros pasaban a diferenciarse de los esnobs al contar con un elenco de gustos más amplio, mientras la categoría de los unívoros se mantenía como antes. Por tanto, es en 2005 cuando Peterson enuncia las categorías definitivas de su teorización sobre el omnivorismo, construidas en torno a dos ejes: la distinción entre alta cultura y cultura popular; y la amplitud o extensión de los gustos. Dichas categorías serían las siguientes:

a) En primer lugar, los unívoros de la alta cultura o refinados (highbrow univores), cuyos gustos siguen, más o menos al pie de la letra, los valores culturales elitistas dominantes y creadores de distinción social, fundamentalmente manifestaciones relativamente tradicionales de la alta cultura. Naturalmente, pertenecen a grupos sociales asociados a las clases altas y medias altas. 
b) Los omnívoros de la alta cultura o refinados (highbrow omnivores), "omnívoros" auténticos, que disponen de amplios gustos que oscilan desde lo alta cultura al interés por ciertos elementos de la cultura popular. Son además unos consumidores activos. No por casualidad, suelen proceder asimismo de las clases medias y altas.

c) Los unívoros de la cultura popular (lowbrow univores), los "unívoros" auténticos, cuyo elenco de aficiones es reducido, sus hábitos de consumo pasivos y su gusto es considerado, socialmente, como la esencia del denominado "mal gusto".

d) Finalmente, los consumidores omnívoros de la cultura popular (lowbrow omnivores) forman una "nueva" categoría de consumidores que disponen de un abanico de gustos relativamente amplio, pero en el que la mayoría de los objetos de consumo cultural pertenecen a la esfera de la cultura popular, con pocas incursiones en la alta cultura.

En otras palabras, de acuerdo al discurso omnívoro, la nueva idea de refinamiento en el terreno de la cultura debe estar asociada a la posesión de unos gustos amplios, tanto "legítimos" como "desautorizados" (Peterson 2005; Peterson y Kern 1996). Lo opuesto entonces a ese gusto omnívoro, de clase alta, que es capaz de abarcar todo, sería el gusto unívoro, pasivo y que apenas se interesa por la cultura, propio de las clases bajas (Chan y Goldthorpe 2007). Lo unívoro se referirá a un perfil con un gusto estrecho y muy limitado en opciones, normalmente concentradas en objetos de la cultura popular.

El omnivorismo trae consigo otros fenómenos asociados. Así, según Peterson (2005), además de una cuestión de pura configuración de los gustos culturales, implica una mayor apertura mental hacia los otros y una mayor tolerancia. El omnívoro cultural al que le gusta la ópera, los "spaghetti westerns" y la World music es más susceptible de tener una actitud cosmopolita y menos racista que, por ejemplo, la generación previa de esnobs aficionados a la alta cultura. Coulangeon y Lemel (2007) sugieren que el omnivorismo simplemente encaja mejor en la (supuestamente) más abierta y tolerante sociedad red, además de que un mayor estatus social y un nivel educativo más elevado van de la mano con una mayor tolerancia política y una mayor atención a cuestiones éticas y morales.

Finalmente, Peterson (2005) argumenta que el omnivorismo es un fenómeno realmente característico del siglo XXI, pese a que la tendencia al omnivorismo cultural se lleva desarrollando varias décadas y es factible que, en el futuro, se genere una reversión de dicho avance o incluso su fin. El esnobismo, argumenta, encajaba bien como modelo de jerarquía en el terreno del gusto del siglo XX, pero en la actualidad estamos viviendo un período de eclecticismo en el que se estimula ser cool y cosmopolita. Peterson explica además que los canales de transmisión de la cultura han cambiado también de manera significativa (gracias a internet, la televisión, los teléfonos móviles, etc. todo el mundo puede lograr disfrutar de productos culturales de una forma nunca vista anteriormente). Finalmente, la creciente movilidad social ha permitido que la gente de las clases populares y sus gustos puedan alcanzar los de las capas altas de 
la sociedad: la jerarquía en la sociedad ha cambiado y el nivel educativo en general ha aumentado, de forma que los gustos propios de la cultura popular tienen posibilidades reales de ser más aceptados socialmente y compartir espacios con los de la alta cultura. En las discusiones teóricas más recientes, dicha orientación "omnívora" se ha asociado con fuerza a variables como el género, la edad, el lugar geográfico o el nivel educativo, de forma que se ha teorizado que las personas con mayor nivel educativo, los jóvenes (más que los adultos), los residentes en grandes ciudades o las mujeres (más que los hombres) son más proclives a la adopción del omnivorismo en sus gustos culturales (ver Coulangeon y Lemel 2007; Lizardo 2006). En el caso de las mujeres, ello podría deberse a un deseo de adquirir respetabilidad social en un contexto de discriminación de género, aunque en algunas investigaciones (Warde y Gayo-Cal 2009; López-Sintas y GarcíaÁlvarez 2002) no se han podido confirmar diferencias significativas en el consumo de alta cultura por género.

Desde entonces, ha existido una auténtica explosión en todo el mundo de estudios sobre el omnivorismo, tanto en el terreno de la sociología como en el de los estudios culturales. Entre los más relevantes destacarían los realizados en Australia (Bennett, Emmison y Frow 1999); Holanda (van Eijck 2001); Israel (Katz-Gerro y Shavit 1998); Reino Unido (Chan y Goldthorpe 2007; Warde, Martens y Olsen 1999); Estados Unidos (los trabajos de Peterson, pero también de Bryson 1996 o Lizardo 2006); Suecia (Bihagen y Katz-Gerro 2000); Finlandia (Purhonen, Gronow y Rahkonen 2009) 0 España (Ariño 2007; López-Sintas y García-Álvarez 2002 y 2004), entre otros. Como podemos comprobar, la aplicación de estos análisis se ha realizado en sociedades con gran desarrollo económico y un sistema sociopolítico que combina democracia liberal y economía de mercado. La metodología utilizada en estas investigaciones ha sido, habitualmente, la cuantitativa, con la realización de encuestas sobre gustos sobre cuyos datos se han aplicado técnicas estadísticas como análisis de regresión (múltiples y logit) y análisis de clusters. El omnivorismo se ha medido desde dos perspectivas: por un lado, a través de la construcción de líneas escalares de conocimiento, preferencias o bienes que nos gustan (por ejemplo, Bryson 1996); por otro, diseñando tipologías de consumidores con el fin de comparar las combinaciones de su portfolio de aficiones y medir la amplitud de sus gustos (por ejemplo, van Eijck 2001).

Los estudios empíricos a escala nacional antes citados han aportado nuevos elementos al debate. Una de estas cuestiones ha sido la noción de voracidad cultural, asociada a la figura de un consumidor cultural voraz. Esta voracidad cultural es "una dimensión cuantitativa del consumo de ocio basada tanto en el ámbito como en la frecuencia de la participación" (Sullivan y Katz-Gerro 2007). La voracidad se refiere, de forma literal, a alguien hambriento, insaciable, glotón; un consumidor cultural voraz sería así alguien que consume una gran cantidad de objetos culturales de forma frecuente y apasionada. Este tipo de voracidad se asocia, como el omnivorismo, a las personas con un elevado estatus social; Sullivan y Katz-Gerro lo perciben como un indicador de estatus simbólico asociado con, entre otras cosas, estar ocupado, realizar tareas múltiples y poseer unos patrones de consumo que abarcan un amplio rango de objetos. En otras 
palabras, la voracidad se concibe como una cualidad ideal entre los consumidores del capitalismo (pos)moderno, si asumimos que el objetivo fundamental de éste es que los individuos, por encima de todo, consuman (Alonso 2005).

Otro debate reciente ha sido el relacionado con nuevas figuras de consumidor cultural a añadir a las aportadas por Peterson de omnívoros y unívoros, y desarrolladas en un importante artículo firmado por Chan y Goldthorpe (2007). Estos autores han estudiado el consumo cultural en el Reino Unido a partir de la encuesta Arts in England Survey complementada con entrevistas individuales, y sugieren que, a las categorías de omnívoros y unívoros, se deben añadir otras dos más. En primer lugar, a los "inactivos": existe, de acuerdo a su investigación, un grupo nada despreciable de individuos que no están interesados en prácticamente ningún tipo de manifestación ni objeto cultural, y que no consumen nada en este apartado. La existencia de este grupo ha sido confirmada por otras investigaciones (López-Sintas y García-Álvarez 2002). Por otro lado, Chan y Goldthorpe descubren que existe un grupo más amplio que participa del consumo cultural "de vez en cuando", dependiendo del tipo de objetos culturales que estén disponibles en ese momento. Chan y Goldthorpe los van a denominar "paucivoros" (paucivores), un término que se está utilizando en la actualidad con mucha frecuencia en los estudios sobre omnivorismo cultural más recientes. Los paucívoros forman un grupo considerablemente grande, de hecho mayor que el de los propios omnivoros; ambos, paucívoros y omnívoros, pertenecen a estratos sociales más elevados que los miembros de la categoría de inactivos. Su argumento principal, además de la introducción de estas nuevas categorías, es que los omnívoros de su estudio no procedían de la élite social, sino más bien de una clase media (profesores, etc.) para los que el omnivorismo es más un síntoma de buena voluntad que un gusto real modelado en el disfrute la alta cultura. La búsqueda, no obstante, de nuevas categorizaciones más precisas de los consumidores culturales se ha convertido en la base de las últimas publicaciones en la materia. Así, la variable edad se ha considerado a menudo: Coulangeon (2005) cita por ejemplo a los "jóvenes omnívoros"; van Eijck (2001), por su parte, habla de "nuevos omnivoros". Bellavance (2008) considera que la introducción de un eje "nuevo-antiguo" es esencial para profundizar en la distinción, casi más que la existente entre gusto elitista y popular. Así, indica que podemos, en el terreno musical, hablar de cuatro categorías: clásico, de gusto elitista, pero a la vez tradicional, "antiguo"; contemporáneo, de gusto elitista pero prestando más atención a las novedades, a lo "nuevo"; folk, de gusto popular y bastante tradicional; y pop, de gusto popular pero más moderno. El omnívoro cultural combina estas cuatro categorías de maneras muy diversas, dando lugar a distintos tipos de omnívoros. Peterson, en uno de sus últimos trabajos, coincidía en que se puede establecer una diferencia entre los omnívoros culturales tomando como criterios la amplitud del gusto y el nivel del gasto (Peterson y Rossman 2008).

\footnotetext{
${ }^{6}$ El omnivorismo cultural, además, podría además vincularse a otras dimensiones del consumo, como prácticas relacionadas con la posesión de objetos (cámaras fotográficas, medios para reproducir música) que
} 


\section{¿LA DESAPARICIÓN DE LAS JERARQUÍAS CULTURALES? CRÍTICAS Y LIMITACIONES DE LOS ESTUDIOS SOBRE OMNIVORISMO CULTURAL}

Esta teoría del omnívoro cultural se ha planteado, generalmente, como una crítica a la teoría de la distinción establecida por Bourdieu en sus trabajos más clásicos. Para van Eijck (2000), una de las aportaciones esenciales de las investigaciones de Peterson es la de introducir más complejidad en el análisis de los gustos, de forma que la clase social no sea el único y exclusivo factor explicativo. Frente a la idea de diferenciación y jerarquización de los estilos de vida basada en la adquisición de capital cultural que enunciaba Bourdieu, los defensores del concepto de omnivorismo cultural defienden por el contrario que los gustos legítimos de las nuevas clases dominantes se caracterizan, en la actualidad, por un amplio abanico de preferencias culturales, con gustos que se extienden desde las artes más refinadas a manifestaciones propias de subculturas populares. Dicha transformación llega hasta el punto de afirmar que, en la actualidad, la cultura clásica legítima ha perdido su vigencia, siendo sustituida por una mezcolanza de aficiones considerada, en cierto modo, más democrática. La teoría del omnivorismo cultural sería, por tanto, más adecuada para una sociedad plenamente sumergida en la denominada posmodernidad, en la que las jerarquías se estarían difuminando a favor de una mayor individualización.

Sin embargo, esta aproximación juega, en ocasiones, con una visión excesivamente complaciente de la denominada posmodernidad, similar a la planteada por sociólogos como Lipovetsky o Maffesoli y en la que las estructuras jerárquicas, burocráticas e ideológicas de la modernidad dejan paso a una sociedad más individualista, heterogénea y tolerante, sin grandes proyectos ideológicos y en la que el hedonismo, el nomadismo, la identidad, el juego y los proyectos inestables pasan a formar la base de una nueva cultura potmoderna. Ollivier (2008) señala con mucha perspicacia que el discurso dominante actual considera a lo diverso, lo híbrido, lo fluido, lo ecléctico, lo global y lo cosmopolita como valores positivos, frente a otros negativos como lo cerrado, lo unitario, lo homogéneo, lo local, lo estático o lo permanente ${ }^{7}$. La apertura hacia otras formas de expresión (llámese omnivorismo o de otra forma) coinciden en buena medida con llamamientos a adaptarse y ser tolerante, un camino imprescindible para poder enfrentarse al paradigma del cambio, en una sociedad cada vez más heterogénea y sujeta a la sucesión incesante de acontecimientos.

Sin embargo, y de forma paradójica, el diseño de estas investigaciones apenas ha tomado en consideración las dimensiones relacionadas con la identidad, como el género o la etnia, que en raras ocasiones se toman en cuenta, y cuando se hace, siempre

juegan un papel importante en la construcción de la identidad y la interacción con los otros (IImonen 2004).

${ }^{7}$ Discurso no distinto al mantenido por los teóricos del management posmoderno acerca de las características que deben poseer organizaciones y trabajadores en el mercado actual: fluidez, flexibilidad y capacidad de adaptarse al cambio (Alonso y Fernández Rodríguez 2006). 
ha sido como una variable secundaria de control sin profundizar en las diferencias y desigualdades (Ollivier, Gauthier y Truong 2009). En el caso del género, ya investigaciones previas como la de Huyssen (1986) resaltaban diferencias importantes en la conceptualización de lo femenino en los gustos culturales: por ejemplo, en la temprana identificación del "gusto femenino" como masa (el caso de las denominadas "novelas ligeras" del siglo XIX, y muchas otras actuales), o como en los estudios culturales las subculturas juveniles de los chicos se han rotulado como formas de resistencia, mientras que las de las chicas sin embargo no. Un análisis de estas dimensiones más detallado sería de particular interés.

Otras críticas se han centrado en que los análisis de Peterson y otros investigadores del omnivorismo tienden a identificar gustos (o preferencias) con prácticas de consumo, cuando son dos esferas claramente diferenciadas. Por ejemplo, algunas voces (Lahire 2008; Chan y Goldthorpe 2007) han apuntado que "gusto" y "consumo cultural" son, dos cosas distintas, y que los estudios que se han realizado sobre omnivorismo se han centrado esencialmente en el gusto sin medir la actividad cultural real de las personas. Chan y Goldthorpe (2007), entre otros, observan que el omnivorismo cultural no es algo que forme parte de la identidad del homo consomator de los pies a la cabeza: es posible ser un verdadero omnívoro cultural en lo que se refiere a la música, pero la misma persona no tiene que serlo en relación a, por ejemplo, los programas de televisión. Otros autores, como van Rees, Vermunt y Verboord (1999) han señalado que el omnivorismo no debería ser medido únicamente a partir de un sumatorio del número de estilos preferidos dentro de cada sector cultural (ópera, ballet, pop-rock, etc.), sino que sería más importante conocer el número de ítems culturales que cada consumidor disfruta (dentro de cada uno de esos estilos), y la forma en que dichos estilos e ítems son estratificados de acuerdo a una escala de legitimidad social. Estos autores señalan además la importancia de realizar estudios longitudinales para comprobar la variación de los gustos de los encuestados a lo largo del tiempo, e incluir el análisis de otras prácticas y preferencias culturales, no centrándose solamente en la música. Y es que un aspecto clave aquí es el excesivo peso que se da a esta última en estas investigaciones, en detrimento de otras áreas de la cultura como el cine, la pintura o el teatro. Como apunta también Lahire (2008), Peterson y sus colegas sólo utilizan datos sobre las preferencias musicales para diseñar sus investigaciones, pero ¿cómo cambiarían los resultados si lo que se utilizase como núcleo de la encuesta fuese otro campo de la cultura o la participación cultural efectiva? De hecho, la investigación de Lahire es más amplia y ambiciosa, al centrarse en los denominados "estilos de vida culturales", y registrando las variaciones de los gustos de los individuos tanto entre los distintos dominios de la cultura, como en el ámbito interno de dichos dominios.

Existe además, en nuestra opinión, una gran dificultad en atribuir elitismo 0 vulgaridad a determinadas categorías musicales de forma adecuada. Por ejemplo, si pensamos en los cuestionarios referidos a gustos musicales, las categorías "jazz" o 
"pop-rock" pueden ser muy poco descriptivas en ese sentido. La categoría "jazz", por ejemplo, se considera en algunas investigaciones (Lizardo y Skiles 2009) como estilo no elitista, minusvalorando las importantes diferencias estilísticas de los artistas y estilos que forman parte del mismo: no es lo mismo la música de big bands tipo Glenn Miller 0 el jazz vocal que las exploraciones sonoras de artistas del free-jazz como Alice Coltrane o Pharoah Sanders, ejecutores de piezas más complejas y poco comerciales. Así, se pasan por alto tanto diferencias en la exigencia requerida por parte del oyente como las distintas representaciones de los estilos en el universo social (los discos de Glenn Miller se venden en grandes almacenes en ediciones poco cuidadas y a precios bajos, los de free jazz se comercializan en tiendas pequeñas independientes y restauran en lo posible las ediciones originales). Lo mismo cabe decir del estilo pop-rock, ya que son prácticas muy distintas escuchar música de las emisoras de radio comerciales y coleccionar álbumes de psicodelia, rock progresivo o post-punk, existiendo además importantes diferencias en la catalogación del gusto en este campo (Bennett, 2009). ¿Es equivalente el grado de legitimidad que tiene la música de la cantante Mariah Carey con la del grupo de rock alemán The Can en los medios especializados? No exactamente, y sus públicos son muy distintos, no tanto quizá en origen social como en las trayectorias de conformación de su gusto personal. No es casual que, en el estudio cualitativo realizado por Bellavance (2008), el entrevistado más elitista era, curiosamente, el más aficionado a escuchar música pop. Asimismo, el dejar de lado otras actividades culturales (teatro, asistencia a exposiciones y conferencias, etc.) impide realizar una adecuada valoración de las prioridades que se adoptan en contextos culturales distintos: como Lahire (2008) apunta, es interesante notar que actividades como escuchar la radio o ver la televisión se realizan en la intimidad del hogar y se caracterizan por ser gratuitas, con lo que las pautas de consumo cultural de estos formatos variarán con respecto a otras en las que se exija una asistencia a un lugar público (siendo así vistos por los demás) y cuesten un precio.

Como se ha comentado, son escasos los estudios en otros campos de la producción cultural, y los pocos que se han realizado han llamado la atención acerca de factores que no se encuentran necesariamente presentes en el terreno del consumo de productos musicales. Por ejemplo, Lizardo y Skiles (2009) señalan que una dimensión poco tenida en cuenta es la del propio contexto de la producción cultural, y que es de enorme importancia: en su análisis comparado sobre gustos televisivos en Europa observaron que en algunos países las distinciones eran mucho menores que en otros, pero el factor clave de esas diferencias entre elitismo y gusto popular venía determinado por los programas de televisión realmente disponibles en cada país y de la calidad de las programaciones de sus cadenas (mientras el acceso a estilos diferentes de música suele ser más sencillo). Lahire (2008) también se ha interesado por el contexto de producción cultural, al afirmar que en la actualidad estamos asistiendo a una crisis de la alta cultura legítima ante el ascenso de grandes compañías del sector privado, las cuales pretenden rentabilizar el mercado de los productos culturales. Finalmente, la producción cultural parece también fundamental en la medición de aquellas actividades que exijan 
una asistencia física para disfrutar de la experiencia cultural: así, en el caso del cine, el teatro o las exposiciones culturales, su visualización depende en buena medida de la posibilidad de acceso a las salas: no es extraño que, en ciudades de provincias, omnívoros culturales o esnobs tengan dificultades para cultivarse en esos ámbitos, pese a que sí puedan disponer de acceso a otras actividades como el disfrute de música 0 literatura (en el hogar). Warde y Gayo-Cal (2009), por ejemplo, han encontrado en su análisis del omnivorismo en el Reino Unido grandes diferencias entre vivir en Londres y en el resto de las ciudades.

Tampoco se han tomado en cuenta otros elementos que influirían en la participación cultural. Por ejemplo, Kraaykamp, van Gils y Ulter (2008) sostienen que el trabajo a tiempo completo o parcial ejerce gran influencia en la participación en actos culturales. Así, el hecho de que los dos miembros de la pareja trabajen a tiempo completo parece retraerlos notablemente de la asistencia a exposiciones y otros actos culturales (según los autores, porque existe una priorización, por parte de los dos miembros de la pareja, a asistir juntos), mientras que si el trabajo de al menos uno de ellos es a tiempo parcial, la participación crece significativamente, sobre todo la de los hombres. Esto demuestra que existen factores importantes, como por ejemplo el tiempo de ocio real disponible, que influyen en el consumo cultural y que poco tienen que ver con la posesión de un gusto omnívoro. Vinculado a esta cuestión, se echa también de menos el estudio de pautas relacionadas con los viajes al extranjero y el consumo cultural en los mismos, que podría proporcionarnos datos muy significativos en relación a las pautas de consumo y estrategias de distinción entre omnívoros y unívoros.

También es necesario hacer una referencia a la metodología de estas investigaciones, basada esencialmente, como ya habíamos comentado, en análisis cuantitativos sobre encuestas de mayor o menor tamaño, salvo raras excepciones. Esto presenta algunas limitaciones. En ocasiones, la utilización de los datos en estos estudios cuantitativos puede generar problemas. Por ejemplo, y como critica Wuggenig (2007), en ocasiones la utilización de datos secundarios (por ejemplo, encuestas de ministerios y organismos oficiales) puede impedir una adecuada categorización de los entrevistados, recomendando que siempre se preparen cuestionarios específicos para este tipo de investigaciones. Algunos elementos en la formulación de los cuestionarios puede inducir a minusvalorar la estratificación cultural real. En muchos casos las preguntas son excesivamente generales: por ejemplo, la investigación de López-Sintas y GarcíaÁlvarez (2002) se centra en una pregunta de la encuesta de CIRES que reza así: "¿ha acudido a alguna de las siguientes actividades culturales en los últimos doce meses?". A nuestro juicio, y como se ha podido observar en la discusión aquí presentada, el concepto de omnivorismo es quizá demasiado complejo como para captar todas sus dimensiones en un trabajo de campo basado en esta pregunta. Ollivier (2008), por su parte, critica que las preguntas de los cuestionarios tienden a ser construidas a través de la óptica de la cultura legítima, con lo que es relativamente fácil que las clases bajas, al no encontrar manifestaciones culturales populares suficientes, queden retratadas como unívoras, siendo muy difícil medir su verdadero grado de apertura cultural. 
Por otra parte, centrarse en una metodología puramente cuantitativa supone importantes limitaciones. En nuestra opinión, parece evidente la necesidad de incorporar metodologías cualitativas en este tipo de investigaciones, pues pueden proporcionar valiosos datos empíricos que pueden contribuir a iluminar importantes aspectos de este interesante debate; entre otros, una mejor captación del elemento elitista y diferenciador en el gusto, a través de un análisis del discurso sobre el mismo. Por ejemplo, Ollivier (2008) o Lahire (2008) consideran que es fundamental el análisis de los discursos de los consumidores, sobre todo en el plano de las justificaciones de los gustos propios; permite además recoger detalles sobre las trayectorias personales de los individuos y los contextos sociales en los que se generan los gustos. La aplicación de técnicas cualitativas se está extendiendo recientemente en los análisis sobre omnivorismo, particularmente las entrevistas, aunque se ha llegado a utilizar en algunas de ellas la técnica de la observación (Bellavance 2008) o incluso se está planteando la utilización de los grupos de discusión que, no debemos olvidar, ha jugado un importante desarrollo en los estudios sobre consumo y la investigación de mercados y audiencias (Callejo 2001; Alonso y Fernández Rodríguez 2007). Algunas de las escasas aplicaciones de estas técnicas cualitativas han dado lugar a resultados de enorme interés, como veremos a continuación.

Las investigaciones del equipo británico coordinado por Alan Warde son un buen ejemplo de una sabia combinación entre métodos cuantitativos y cualitativos, pues consideran esencial la realización de entrevistas para poder captar las justificaciones y motivaciones de los omnívoros. De hecho, las ocho entrevistas realizadas en Warde, Wright y Cayo-Gal (2007) les sirven para detectar diferencias significativas entre los propios omnívoros, que les lleva a clasificarlos en cuatro categorías: los profesionales, que trabajan en sectores cercanos a la producción cultural; los disidentes, cuya actitud de fondo es una rebeldía ante los valores culturales convencionales; los aprendices, una categoría no detectada por Peterson de personas con escaso capital cultural pero una sorprendente capacidad de estar al tanto de algunas de las nuevas tendencias en arte, normalmente por el deseo de experimentar dentro del contexto de una gran metrópoli; y los sencillos o sin pretensiones (unassuming), que representan los gustos de la clase media sin asumir riesgos. Otro ejemplo de utilización de la entrevista es el trabajo de Ollivier (2008), en el que las utiliza como complemento a una encuesta, $y$, sin duda, los resultados de dichas entrevistas son lo suficientemente ricos como para reformular las categorías con las que se estaba discutiendo el concepto de omnivorismo. De este modo, en lugar de omnivorismo prefiere hablar de maneras de apertura a la diversidad cultural, de las que existirían cuatro tipos: una forma humanista, caracterizados como consumidores de cultura muy activos, críticos con la cultura de masas, elitistas pero abiertos a la incorporación irónica de elementos de la cultura popular (a veces de una forma no tan irónica); otra populista, con gente de cultura media, que trata de mostrar buena voluntad a la hora de aproximarse a la alta cultura y que enfatiza la idea de relajación cuando habla de las razones por las que consume cultura; otra forma de apertura es la práctica, común entre hombres de clase 
obrera, en la que la buena voluntad no es perceptible y la apertura cultural tiene que ver con un cierto sentido práctico; y finalmente, una forma indiferente, propia también de las clases bajas, en la que no se manifiesta interés ni conocimientos en relación al mundo de la cultura. Los resultados de estas investigaciones concluyen en que las desigualdades socioeconómicas tienen una importante influencia en el consumo cultural, permaneciendo todavía vigentes las jerarquías culturales.

Y es que ésta es precisamente una de las razones por las que más se ha criticado esta teoría del omnivorismo: su juicio implícito de que las diferencias de clase se están difuminando, en correspondencia con análisis que anuncian la desaparición progresiva de la sociedad de clases (por ejemplo, Pakulski y Walters 1996). Varias investigaciones certifican que la distinción y la jerarquización social de los gustos culturales siguen todavía vigentes. Ya en un artículo muy influyente sobre los estilos musicales que menos agradaban a los norteamericanos, Bryson (1996) hacía notar que el aparentemente "amplio" gusto de aquellos con mayor nivel educativo sólo lo es en el sentido de que existen muchas cosas que les gustan; pero llamativamente, los géneros musicales cuyos aficionados se caracterizan por tener un menor nivel educativo (góspel, country, rap o heavy metal) suelen ser aquellos mayoritariamente rechazados por los que se consideran musicalmente tolerantes. Así, puede argumentarse que el aparentemente tolerante gusto de los omnívoros acaba rechazando, de forma feroz, aquellos elementos culturales asociados de forma inequívoca a las culturas populares, lo que muestra, sin lugar a dudas, que incluso en el omnivorismo las barreras de la exclusividad y la distinción siguen operando de alguna forma. De hecho en otras investigaciones se ha observado que en las entrevistas, por ejemplo, algunos individuos de clases medias y bajas se sentían un tanto inseguros y reacios a hablar de sus competencias culturales, frente a la seguridad con la que se expresaban los miembros de las élites (Silva y Wright 2005). Este tipo de situaciones ejemplifican la forma en que actúa la jerarquía cultural, mostrando además detalles que una investigación puramente cuantitativa no puede reflejar.

No obstante, en nuestra opinión, la crítica más completa al trabajo de Peterson es quizá la de Bernard Lahire. En contraste con las teorías de Bourdieu y, en parte, con las de Peterson, Lahire $(2004 ; 2008)$ sugiere que, en lugar de gustos basados en la clase social, o gustos de omnívoros versus gustos de unívoros, deberíamos hablar de un abanico de gustos individuales que puede ser consonante o disonante respecto al grupo social de referencia. Ser consonante significa mantener una coherencia en las preferencias y prácticas culturales respecto al grupo de referencia, y este grupo puede poseer un gusto tanto socialmente legítimo (elitista, caso de las clases altas) como ilegítimo (popular, caso de las clases bajas); ser disonante implica precisamente que las preferencias y prácticas culturales del individuo difieren o son atípicas respecto al grupo social de referencia. Para Lahire, por tanto, las disposiciones en el terreno del gusto (tanto al nivel de las preferencias como de las prácticas reales) son individuales, pues afirma que, si bien es cierto que los individuos tienden a comportarse en gran medida como el resto de su grupo social, también lo es que en algunos aspectos de su 
comportamiento, serán atípicos respecto a dicho grupo social de referencia, y de hecho las estadísticas confirman que esto es lo que sucede con más frecuencia. Defiende así la importancia del contacto con los otros (de otras clases sociales, de otros grupos) en la conformación de auténticas personalidades individuales, atravesadas e influidas por lo social, pero estando esta idea de lo social caracterizada por la heterogeneidad y la diversidad. Lahire afirma que nada es más estadísticamente frecuente que la singularidad individual, lo que le lleva a afirmar que la disonancia es más la norma que la excepción en la sociedad contemporánea.

Así, los gustos van a variar desde lo más refinado a lo más popular influidos no únicamente por el origen de clase, sino también por la exposición a influencias socializadoras heterogéneas (los amigos, la educación o la profesión) resultado en buena medida de la movilidad educativa, social y profesional. Las legitimaciones en los diferentes campos de la sociedad están así continuamente reformuladas y re-producidas. Según Lahire (2008), el gusto es un abanico que variará a lo largo del tiempo y en diferentes fases de la vida del individuo, ya que en las sociedades actuales los individuos cambian a lo largo de la vida de grupo de referencia. No se puede establecer así una mera división entre omnívoros (que consumen numerosos productos culturales sin situarlos en lugar alguno dentro de la jerarquía) y unívoros (que sólo consumen un rango limitado de productos culturales, normalmente dentro de la esfera de la cultura popular): centrarse en perfiles consonantes y disonantes recogerá la esencia del consumidor moderno.

Lahire realiza una elaborada crítica a Peterson, fundamentalmente en el aspecto metodológico. Más allá del comentario antes citado sobre la necesidad de expandir la investigación de la música a favor de un más amplio "estilo de vida cultural", el sociólogo francés indica que las investigaciones de Peterson solamente recogen datos acerca de las preferencias musicales, dejando de lado la esfera de las prácticas culturales en este dominio, "prácticas que no siempre se eligen". Así, en muchos casos, aunque exista una preferencia, la práctica real puede ser el resultado de un compromiso con la pareja, un familiar o un amigo, en forma de elección "acompañada". Asimismo, algunas de estas prácticas están marcadas por ciertos automatismos que las hacen muy diferentes a preferencias elegidas en un contexto de acción racional: los gustos no son individuales sino que se forman mediante una combinación entre las disposiciones internas del individuo y el contexto externo en el que realiza sus prácticas.

Para Lahire, todas las clases sociales participarían así del eclecticismo: por ello, se muestra muy crítico con el concepto de omnivorismo, al achacarle que en el mismo sólo las clases altas son presentadas como culturalmente eclécticas. En este sentido,

\footnotetext{
${ }^{8}$ Son posibles otras categorizaciones relacionadas con la manera de disfrutar o acercarse a la cultura: lo podemos hacer solos (entendiendo la cultura como un fin en sí mismo: asistir solo a una sala de cine de autor, por ejemplo), o en compañía (entendiendo la cultura como medio o pretexto para establecer una relación social), y formas distintas de apropiarnos de ella (comprar o descargar de páginas web en internet).
} 
la crítica que aquí se formula al omnivorismo cultural es similar a la que se ha realizado a la teoría de Bourdieu: en ambas parece que son las clases altas las únicas que se muestran como activas, curiosas y abiertas a nuevas experiencias; mientras que las clases bajas aparecen como pasivas ante la cultura y despreocupadas por su estilo de vida. Además, en el caso de Peterson se crea una imagen de unas clases altas tolerantes y abiertas frente a unas clases bajas intolerantes que, a su juicio, es falsa; ya que Peterson sostiene este argumento sobre la suposición, muy ingenua, de que el disponer de unos gustos relativamente amplios o abiertos implica de forma automática una actitud tolerante hacia los demás. En cierto sentido, la teoría del omnivorismo cultural podría ser catalogada, desde esta perspectiva, como cargada de un cierto elitismo. Ollivier (2008) comparte esta crítica en gran medida, ya que contar con un abanico de gustos amplio y ser tolerante hacia los demás no queda demostrado.

Finalmente, otro trabajo destacable es el de Colulangeon y Lemel (2007), que han señalado que, en Francia, los datos empíricos relacionados con los hábitos de audición de música corroboran la teoría de Peterson del omnivorismo cultural, e invalidan así la estricta homología establecida por Bourdieu. Ya no sería posible establecer esa correspondencia unívoca entre un género musical y una clase social concreta que confirmaría la tesis de la distinción a través del gusto musical, aunque señalan que esto únicamente confronta parcialmente la teoría de Bourdieu, ya que efectivamente el gusto omnívoro se concentra en las clases altas: ello confirma que existe una estratificación social de los gustos. El motivo por el que las clases altas se convertirían en omnívoras sería, en primer lugar, porque gracias a la movilidad social fruto de la escolarización masiva, nuevos grupos con experiencias vitales más heterogéneas y procedencias de clase distintas se están incorporando a las élites sociales, lo que fragmenta los gustos; y por otra parte, porque el eclecticismo se ha convertido, debido a la mayor valoración social que ha adquirido la apertura y la tolerancia, en una nueva herramienta para las estrategias de distinción, diagnóstico con el que coincide Ariño (2007). Por lo tanto, parece que la distinción sigue gozando de buena salud.

\section{Conclusiones}

Como se ha podido comprobar en este artículo, el debate sobre el omnivorismo cultural es uno de los más activos en la sociología del consumo contemporánea. El argumento fundamental de los defensores de esta teoría (Peterson y colaboradores) es que los consumidores refinados, que suelen formar parte de una élite social (por su clase social, nivel educativo e incluso raza) desde hace varias décadas están mostrando una mayor variedad de gustos y atendiendo a manifestaciones culturales (exposiciones, conciertos) más diversas que los consumidores culturales no refinados. Este abanico de gustos más amplio va de la mano con una actitud general supuestamente más abierta y tolerante, de forma que las élites y las clases medias altas serían, por ejemplo, menos racistas que las clases bajas. De esta forma, en Estados Unidos los antiguos esnobs de clase 
alta estarían siendo reemplazados, progresivamente, por cohortes más jóvenes de consumidores caracterizados como omnívoros en sus gustos culturales.

Este concepto de omnivorismo cultural ha servido para impulsar un gran número de investigaciones empíricas cuyos resultados han servido no sólo para estimular la creación de nuevos términos en el ámbito sociológico (los conceptos paucívoro o consumidor cultural voraz son solo dos ejemplos), sino también para lanzar una discusión crítica. Así, se ha señalado un conjunto de asuntos que cuestionan los argumentos presentados por Peterson y colaboradores: por ejemplo, que la amplitud del abanico de gustos puede ser una nueva forma de distinción en el sentido bourdieuano del término, reforzando las barreras simbólicas entre los omnívoros y la gente de clases más bajas (la crítica de Bryson 1996), de forma que la tolerancia hacia los objetos culturales se construye como otra forma de violencia simbólica hacia los miembros de las clases populares. Otras críticas que se han realizado a este concepto, como ya hemos visto, son las de que en realidad mide el gusto y no la participación real en actividades culturales; que el grado de omnivorismo es muy variable entre los diferentes campos culturales; que Peterson y sus colaboradores sólo toman en cuenta el campo de la música; y que las disposiciones relacionadas con el gusto varían lo largo de la trayectoria vital de los individuos, pudiendo metamorfosearse de unívoro a omnívoro y de gusto refinado a gusto popular, de acuerdo a la socialización, la familia, el lugar de trabajo, etcétera. Aunque en la obra de Peterson se hagan referencias continuas al ascenso del individualismo, la agencia y la reflexividad, no cabe duda de que en la relación de los individuos con la cultura todavía perviven importantes jerarquías, resultado de la organización desigual de las posiciones sociales.

Es cierto que es difícil definir la legitimidad cultural hoy en día, en un escenario de creciente fragmentación del consumo cultural no ajeno a la tantas veces denunciada fragmentación social del posfordismo (Alonso 2006). El omnivorismo, como se ha defendido por parte de algunas posiciones de este debate, no parece exclusivo de las clases altas. $Y$ sobre todo no supone el fin de las barreras culturales ni de las jerarquías: los mecanismos de exclusión continúan operando, aunque ahora basados en una pluralidad de diferencias más compleja. Ante este escenario, parece que se hace necesario seguir profundizando en el estudio sobre el fenómeno de la distinción, desarrollando más estudios que enfaticen la dimensión comparativa y que tengan en cuenta la influencia de las condiciones sociohistóricas. Quizá la introducción de análisis cualitativos pueda ser beneficiosa para captar algunas de sus complejidades. En el caso de España, esta última tarea aún no se ha acometido, pese a contar con una tradición relevante de estudios cualitativos en sociología del consumo con la que podrían entroncarse. 


\section{REFERENCIAS BIBLIOGRÁFICAS}

Alonso, L. E. 2005. La era del consumo. Madrid: Siglo XXI.

Alonso, L. E. 2006. "A Post-Fordist Consumption Norm? Social Fragmentation, Individualization and New Inequalities". Pp. 153-168 en Employment Relations in a Changing Society. Assesing the PostFordist Paradigm, edited by L. E. Alonso y M. Martínez Lucio. Basingstoke: Palgrave Macmillan.

Alonso, L. E. y C. J. Fernández Rodríguez. 2006. "El imaginario 'managerial': el discurso de la fluidez en la sociedad económica", Política y Sociedad 43:127-151.

Alonso, L. E. y C. J. Fernández Rodríguez. 2007. "Sociología del consumo". Pp. 455-480 en La sociología en España, editado por M. Pérez-Yruela. Madrid: CIS.

Alonso L. E., E. Martín Criado y J. L. Moreno Pestaña. eds. 2004. Pierre Bourdieu, las herramientas del sociólogo. Madrid: Fundamentos.

Ariño, A. 2007. "Música, democratización y omnivoridad." Política y Sociedad 44 (3):131-150.

Bellavance, G. 2008. "Where's High? Who's Low? What's New? Classification and Stratification inside Cultural Repertoires." Poetics 36 (2-3):189-216.

Bennett, A. 2009. "'Heritage Rock': Rock Music, Representation and Language Discourse." Poetics 37 (5-6):474-489.

Bennett, T., M. Emmison y J. Frow. 1999. Accounting for Taste: Australian Everyday Cultures in the Life of Australians. Cambridge: Cambridge University Press.

Bergham, M., y K. Van Eijck 2009. "Visual Arts and Appreciation Patterns: Crossing Horizontal and Vertical Boundaries within the Cultural Hierarchy." Poetics 37 (4):348-365.

Bihagen, E, y T. Katz-Gerro. 2000. "Cultural consumption in Sweden: the stability of gender differences." Poetics 27(5-6):327-349.

Bourdieu, P. 1983. "The Field of Cultural Production, or the Economic World Reversed." Poetics 12 (4-5):311-356.

Bourdieu, P. 1998. La distinción. Criterios y bases sociales del gusto. Madrid: Taurus.

Brooks, D. 2001. BoBos en el paraíso. Barcelona: Mondadori.

Bryson, B. 1996. "'Anything but Heavy Metal': Symbolic Exclusion and Musical Dislikes", American Journal of Sociology 102(3):884-899.

Callejo, J. 1995. "Elementos para una teoría sociológica del consumo." Papers 47:75-96.

Callejo, J. 2001. Investigar las audiencias. Un análisis cualitativo. Barcelona: Paidós.

Chan, T. W. y J. H. Goldthorpe. 2007. "Social Stratification and Cultural Consumption: The Visual Arts in England." Poetics 35 (2-3):168-190. 
Coulangeon, P. 2005. "Social Stratification of Musical Tastes." Revue Française de Sociologie 46 (2-3): 123-154.

Coulangeon, P. e Y. Lemel. 2007. "Is 'Distinction' Really Outdated? Questioning the Meaning of the Omnivorization of Musical Taste in Contemporary France." Poetics 35:93-111.

Daloz, J. P. 2009. The Sociology Of Elite Distinction. From Theoretical To Comparative Perspectives. Londres: Palgrave Macmillan.

Di Maggio, P. 1987. "Classification in Art." American Sociological Review 52:440-455.

Douglas, M. y B. Isherwood. 1979. El mundo de los bienes. Hacia una antropología del consumo. México DF: Grijalbo.

Fernández Porta, E. 2008. Homo Sampler. Tiempo y consumo en la era afterpop. Barcelona: Anagrama.

Florida, R. 2002. The Rise of the Creative Class: And How It's Transforming Work, Leisure, Community and Everyday Life. Nueva York: Basic Books.

Hannerz, U. 1990. "Cosmopolitans and Locals in World Culture." Theory, Culture \& Society 7(2): 237251.

Harvey, D. 1998. La condición de la posmodernidad: investigación sobre los orígenes del cambio cultural. Buenos Aires: Amorrortu.

Huyssen, A. 1986. "Mass Culture as Woman: Modernism's Other", Pp. 44-62 en After the Great Divide, edited by A. Huyssen. Bloomington, IN: Indiana University Press.

IImonen, K. 2004. "The Use of and Commitment to Goods." Journal of Consumer Culture 4(1): 27-50.

Kane, D. 2003. "Distinction Worldwide? Bourdieu's Theory of Taste in International Context." Poetics 31(5): 403-421.

Katz-Gerro, T. e Y Shavit. 1998. "The Stratification of Leisure and Taste: Classes and Lifestyles in Israel." European Sociological Review 14(4): 369-386.

Kraaykamp, G., W. van Gils y W. Ulter. 2008. "Cultural Participation and Time Restrictions: Explaining the Frequency of Individual and Joint Cultural Visits." Poetics 36(4): 316-332.

Lahire, B. 2004. La Culture des Individus: Dissonances Culturelles et Distinction de Soi. París: Editions La Decouverte.

Lahire, B. 2008. "The Individual and the Mixing of Genres: Cultural Dissonance and Self-Distinction." Poetics 36(2-3): 166-188.

Lamont, M. 1992. Money, Morals and Manners. Chicago: Chicago University Press.

Lizardo, O. 2006. "The Puzzle of Women's 'Highbrow' Culture Consumption: Integrating Gender and Work into Bourdieu's Class Theory of Taste." Poetics 34(1): 1-23. 
Lizardo, O. y S. Skiles. 2009, "Highbrow Omnivorousness on the Small Screen? Cultural Industry Systems and Patterns of Cultural Choice in Europe." Poetics 37(1): 1-23.

López-Sintas, J. y E. García-Álvarez. 2002. "Omnivores Show Up Again: The Segmentation of Cultural Consumers in Spanish Social Space." European Sociological Review 18(3):353-368.

López-Sintas, J. y E. García-Álvarez. 2004. "Omnivore versus Univore Consumption and its Symbolic Properties: Evidence from Spaniards' Performing Arts Attendance." Poetics 32(6): 463-84.

Noya, F. J. 1998. "Omnívoros sociables: consumo y capital relacional en España." Sociológica: Revista de pensamiento social 3:69-92.

Ollivier, M. 2008. "Modes of Openness to Cultural Diversity: Humanist, Populist, Practical, and Indifferent." Poetics 36(2-3): 120-147.

Ollivier, M., G. Gauthier y A. H. Truong. 2009. "Cultural classifications and social divisions: A symmetrical approach." Poetics 37(5-6): 456-473.

Pakulski, J. y M. Walters. 1996. The Death of Class. Londres: Sage.

Peterson, R. A. 1992. "Understanding Audience Segmentation: From Elite and Mass to Omnivore and Univore." Poetics 21(4): 243-258.

Peterson, R. A. 2005. "Problems in Comparative Research: The Example of Omnivorousness." Poetics 33(5-6): 257-282.

Peterson, R. A. y Rossman, G. 2008. "Changing Art Audiences", Pp. 307-342 en Engaging Art, edited by B. Ivey y S. Tepper. Nueva York: Routledge.

Peterson, R. A. y R. M. Kern. 1996. "Changing Highbrow Taste: From Snob to Omnivore." American Sociological Review 61(5): 900-909.

Peterson, R. A. y A. Simkus. 1992. "How Musical Tastes Mark Occupational Status Groups". Pp. 152-186 en Cultivating Differences, edited by M. Lamont y M. Fournier. Chicago: University of Chicago Press.

Purhonen, S., J. Gronow y K. Rahkonen. 2009. "Social Differentiation of Musical and Literary Taste Patterns in Finland." Research on Finnish Society 2: 39-49.

Silva, E. y D. Wright. 2005. "The Judgement of Taste and Social Position in Focus Group Research." Sociologia i Ricerca Sociale 76-77: 241-253.

Sullivan, O. y T. Katz-Gerro. 2007. "The Omnivore Thesis Revisited: Voracious Cultural Consumers." European Sociological Review 23(2): 123-137.

Van Eijck, K. 2000. "Richard A. Peterson and the culture of consumption." Poetics 28(2): 207-224.

Van Eijck, K. 2001. "Social differentiation in musical taste patterns." Social Forces 79(3): 1163-1184.

Van Rees, K., J. K. Vermunt y M. Verboord. 1999. "Cultural classifications under discussion: latent class analysis of highbrow and lowbrow reading." Poetics 26(5-6): 349-365. 
Warde, A. y M. Gayo-Cal. 2009. "The anatomy of cultural omnivorousness: The case of the United Kingdom." Poetics 37(2): 119-145.

Warde, A., L. Martens y W. Olsen. 1999. "Consumption and the problem of variety: cultural omnivorousness, distinction, and dining out." Sociology 33(1): 105-127.

Warde, A., D. Wright y M. Gayo-Cal. 2007. "Understanding Cultural Omnivorousness: Or, the Myth of the Cultural Omnivore." Cultural Sociology 1(2): 143-164.

Wuggenig, U. 2007. "Comments on Chan and Goldthorpe: Pitfalls in testing Bourdieu's homology assumptions using mainstream social science methodology: Social stratification and cultural consumption: The visual arts in England." Poetics 35(4-5): 306-316.

CARLOS JESÚS FERNÁNDEZ RODRÍGUEZ es profesor contratado doctor en el Departamento de Sociología de la Universidad Autónoma de Madrid (UAM). Especializado en sociología de las organizaciones, del trabajo y del consumo, ha publicado hasta la fecha una veintena de artículos y varios libros en torno a estas temáticas.

RIIE HEIKKILÄ es doctora en Ciencias Sociales por la Universidad de Helsinki, donde trabaja como investigadora del departamento de Social Policy. Especializada en sociología del consumo cultural y en estudios sobre la minoría sueco-finlandesa, ha publicado varios artículos en revistas nacionales e internacionales.

RECIBIDO: $15 / 04 / 2010$

ACEPTADO: 10/01/2011 